\title{
Non-HIV pneumocystis pneumonia
}

\author{
Nobuhiro Asai ${ }^{1,2}$ \\ Yoshihiro Ohkuni ${ }^{2}$ \\ Norihiro Kaneko² \\ Shinji Motojima ${ }^{3}$
}

\author{
1 Department of Internal Medicine, Division of Respi- \\ ratory Medicine and Allergology, Aichi Medical Univer- \\ sity School of Medicine, Aichi, Japan \\ 2 Pulmonology Department, Kameda Medical Center, \\ Chiba, Japan \\ ${ }^{3}$ Rheumatology Department, Kameda Medical Center, \\ Chiba, Japan
}

\section{Address for correspondence:}

Nobuhiro Asai

Department of Internal Medicine, Division of Respiratory Medicine and Allergology, Aichi Medical University School of Medicine, Aichi, Japan

Zip 480-1195 1-1 Yazakokarimata, Nagakute, Aichi, Japan

E-mail: nobuhiro0204@hotmail.com

\section{Summary}

Human immunodeficiency virus (HIV)-uninfected Pneumocystis jirovecii pneumonia (non-HIV PCP) can develop in patients with autoimmune diseases, malignancies, and other diseases, and it can lead to potentially lethal respiratory dysfunction showing a high mortality (1-3). Over the past decade, a paradigm shift in the treatment of autoimmune disease such as rheumatoid arthritis (RA) $(4,5)$ and inflammatory bowel diseases (IBD) has been brought about by the introduction of biologics (6-8). While the emergence of innovative biologic agents targeted at specific molecules and pathways in the immune system have altered the clinical course of autoimmune disease patients and improved their quality of lives and social outcomes, increasing incidence of non-HIV PCP have been noticed (4-8). In the field of solid organ transplant recipients and malignancies, the emergence of new generation of immunosuppressive agents, such as rituximab and cytotoxic agents could result in frequent occurrence of non-HIV PCP (9-13). Today, although every clinician could encounter PCP patients, there is no established standard treatment for non-HIV PCP. We review recent topics and some aspects to improve the treatment of non-HIV PCP.

KEY WORDS: pneumocystis jirovecii pneumonia, human immunodeficiency virus, non-HIV PCP, rheumatoid arthritis, $\beta$-D-glucan.

\section{Introduction}

Pneumocystis jirovecii pneumonia (PCP) remains one of the most prevalent opportunistic infections in immunosuppressed patients. Effective prophylaxis of PCP and the availability of highly antiretroviral therapy have reduced the morbidity and mortality of PCP in HIV-infected patients. On the other hand, the risk and the incidence of PCP without HIV infection (non-HIV $\mathrm{PCP}$ ) has increased as the number of patients receiving chemotherapy or immunosuppressive agents has been rapidly growing (1-3).

The mortality of patients with non-HIV PCP is diverse and ranges from 0 to $70 \%$ (1, 3-9), compared with the mortality of HIV-infected PCP patients, which ranges from 10 to $20 \%(1$, $3,10)$. The higher mortality among non-HIV patients has been attributed to severe

The incidence of PCP without HIV infection (non-HIV PCP) has increased as the number of patients receiving chemotherapy or immunosuppressive agents did. lung inflammation $(1,3,5)$, although the exact etiology for these large differences in mortality has not yet been determined. Also, the evidenced-based standard treatments, or the therapeutic strategies, are not established yet. These could lead to doctor's and patients' delays, resulting in a high mortality of non-HIV PCP. We review issues reported in the recent publications and recommend the therapeutic strategy for HIVuninfected PCP.

\section{Clinical manifestations}

Clinical symptoms in non-HIV PCP differ from those in HIV-PCP. While almost all the HIV-infected PCP patients show cough, sputum and dyspnea, these respiratory symptoms are less likely seen in non-HIV PCP patients at initial visit. In the article published in 2014 by Roux et al., cough, sputum and hypoxia were less frequently seen in the nonHIV PCP patients than in those with HIV-PCP except for those with fever at initial visit. On the other hand, CRP was much higher in

The early diagnosis of non-HIV PCP is very difficult for clinicians due to paucity of respiratory symptoms compared with HIVPCP. 
the non-HIV-PCP patients than those with HIV-PCP (14). These suggest that early diagnosis as non-HIV PCP is very difficult for clinicians due to poor respiratory symptoms compared with HIV-PCP. This results in a high mortality of non-HIV PCP patients who were commonly misdiagnosed as bacterial pneumonia in a general medicine ward. It is outstanding that the severity of non-HIV PCP might be underestimated by conventional prognostic guidelines for community acquired pneumonia $(15,16)$. If PCP is suspected, chest CT scan should be performed (Figures 1, 2).

\section{Diagnosis}

Poor diagnosis as non-HIV PCP could contribute to a high mortality. There is no cultural method for $P$. jrovecii (4), thus microscopic findings such as Grocott or Diff-Quik staining are commonly used. Although Diff-Quik is specific and useful, it requires high expertise and has a low sensitivity in the diagnosis of nonHIV PCP (17). P. jirovecii-PCR in induced sputum and BALF are very sensitive in the diagnosis of PCP, however colonialization is commonly seen in $20 \%$ of healthy person and $30 \%$ of patients who have chronic pulmonary diseases (18). $\beta$-D-glucan and KL-6 are one of the most useful tools in the diagnosis of PCP and are widespread (19-22). Tasaka reported that serum- $\beta$-D-glucan is useful in the diagnosis of nonHIV PCP. Its cutoff value of $31.1 \mathrm{pg} / \mathrm{ml}$ had a sensitivity of $92 \%$ and a specificity of $86 \%$ for the diagnosis of non-HIV PCP (23). In another study including 111 AIDS patients described by Watanabe, its cutoff value was $23.2 \mathrm{pg} / \mathrm{ml}$ for the diagnosis of PCP (24). Esteves et al. documented that combination of KL- 6 and $\beta$-Dglucan has a high sensitivity and specificity in the diagnosis of non-HIV PCP (25). While some studies for the diagnostic markers of non-HIV PCP have been reported, we sometimes experience a case of HIV-uninfected PCP with a positive microscopic findings, showing lower than normal level markers. In our study, 10 of 33 non-HIV PCP patients had a definite diagnosis with a positive microscopic findings that showed a $\beta$-D-glucan level of lower of than $31.1 \mathrm{pg} / \mathrm{ml}$. If nonHIV PCP is suspected, empiric therapy should be recommended for a few days, resulting in a good prognosis as mentioned later (16). In general practice, diagnosis

as non-HIV PCP should be
In general practice, diagnosis as non-HIV PCP should be standardized in a positive conventional PCR and high serum level of $\beta$-D-glucan with the compatible clinical symptoms and the radiological findings. standardized in a positive conventional PCR and high serum level of $\beta$-D-glucan with the compatible clinical symptoms and the radiological findings (Figures 1,2). Microscopic findings of $P$. jirovecii is reliable, however they are not necessary in diagnosing PCP due to its low sensitivity and the requirement of expertise as above mentioned.
Recently, loop-mediated isothermal amplification

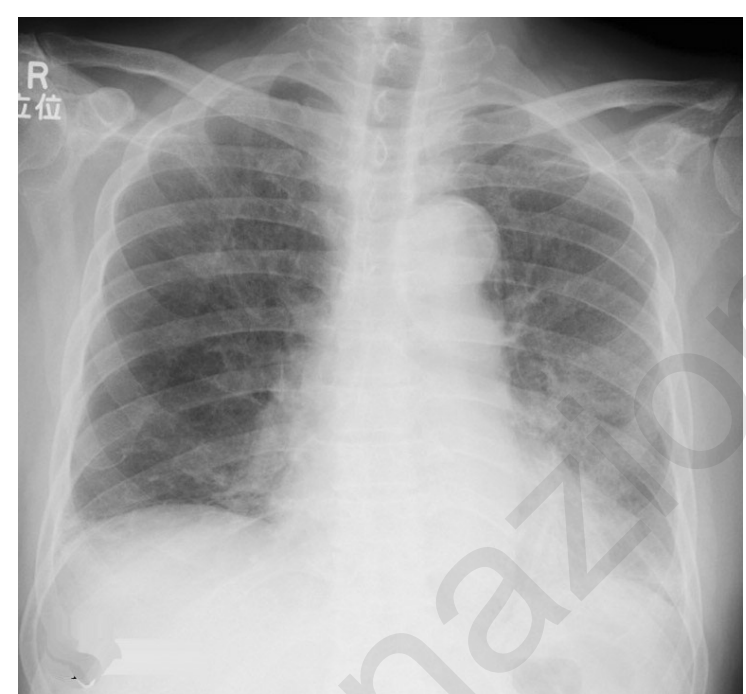

Figure 1 - Chest X-ray shows bilateral reticular shadows in the both lungs.

(LAMP) method for PCP has widely been spread as diagnostic tools for PCP (26-28). While the LAMP method for PCP is a potential diagnostic replacement for PCR, the essence of this method remains the same: it shows nothing except for the existence of either infection or colonization.

The results that LAMP of $P$. jirovecii is positive could just mean a colonization as well as PCR could. The negative results for PCR or LAMP method of $P$. jirovecii are valuable because it means that PCP is not present.

\section{Treatment}

Efficacy of combination therapy of corticosteroid and Sulfamethoxazole/Trimetoprim (TMP-SMX) has been confirmed for HIV-infected PCP. However, there is no evidenced based treatment for non-HIV PCP. The etiology and pathophysiology of non-HIV PCP is quite different from HIV-infected PCP. It is evident that most of the clinical manifestation of PCP is derived from immune response to Pneumocystis jirovecii. Pneumocystis elicits many kinds of immune responses, including those by lymphocytes, macrophages, neutrophils, dendritic cells, and epithelial cells $(1,29)$. Anti-non-HIV PCP therapy must be different from one of HIV-infected PCP. In addition, the association of $P$. jirovecii cyst have been reported to be one-tenth in HIV-uninfected PCP, compared with HIV-infected PCP (3). Therefore, the sensitivity of conventional staining methods for diagnosis of HIV-uninfected PCP is lower than the one for PCP with HIV. Thus, low dose of TMP-SMX should be administered in the treatment of non-HIV PCP once it is suspected, since it works and toxicity of 


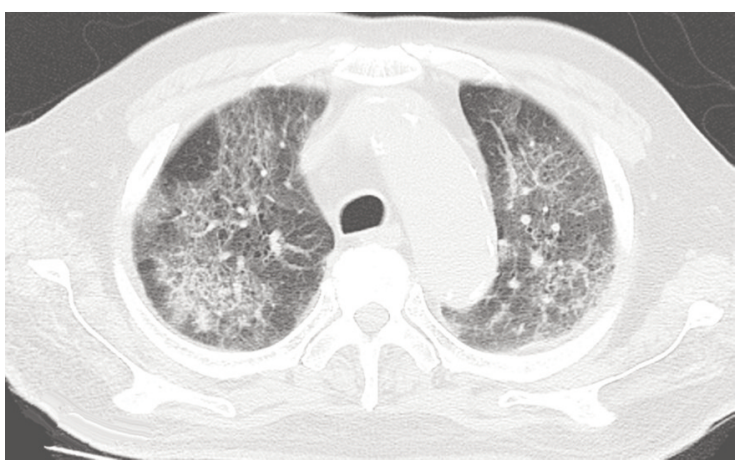

Figure 2 - Chest CT shows panlobular ground-glass opacities in the both lung fields.

TMP-SMX is dose-dependent. Mori documented that combination therapy of corticosteroid and low-dose TMP-SMX is effective and high-dose TMP-SMX is not necessary (18). High-dose TMP-SMX could result in renal failure or volume overload if parenterally administered. In our study, non-HIV PCP patients who had received high-dose of TMP-SMX showed a high mortality compared with those who had received low-dose of TMP-SMX without a statistical difference (50 vs $23.8 \%, p=0.165)$. We recommend that combination of low-dose TMP-SMX (4-6 tablets) with corticosteroid therapy for HIV-uninfected PCP.

\section{Prophylaxis}

Guidelines have been published for the use of PCP prophylaxis in patients with cancer, including hematopoietic stem cell transplant (HCT) recipients (30, 31 ), as well as in solid organ transplant recipients (32). In contrast, there are no published guidelines for $\mathrm{PCP}$ prophylaxis among patients with rheumatologic diseases receiving immunosuppressive drugs. Che-

TMP-SMX is the first
choice prophylaxis
for non-HIV PCP as
well as HIV PCP, and
it is the only agent
showing more effec-
tiveness than place-
bo in prospective
randomized trials
(grade A recommen-
dation).
moprophylaxis has been recommended in patients with rheumatologic diseases such as Systemic lupus erythematosus, Dermatomyositis/ Polymyositis and Wegener's granulomatosis who are treated with significant doses of glucocorticoids ( $\geq 20 \mathrm{mg}$ of prednisone daily for one month or longer) in combination with a second immunosuppressive drug, particularly a cytotoxic agent due to a high incidence of PCP (33-35).

TMP-SMX is the first choice prophylaxis for non-HIV PCP as well as HIV PCP, and is the only agent demonstrated to be more effective than placebo in prospective randomized trials (grade A recommendation) $(36,37)$. The dosage usually recommended is one tablet daily or two tablets three times per week. There was no statistical differences among the regimens for PCP occurrence by a meta-analysis. Ato- vaquone is advocated to be the alternative for $\mathrm{PCP}$ prophylaxis in patients who are not tolerable for TMP. SMX.

\section{Prognosis}

The mortality rate of non-HIV PCP is higher than that of HIV-PCP as mentioned above (1, 3-10). While many studies have reported potential prognostic factors of HIV-PCP, few studies evaluated risk factors for non-HIV PCP. Some documented that high A-aDO2, combined bacteremia, elevated BUN and preexisting lung disease were independently poor prognostic factors $(38,39)$. Also, it has been reported that early diagnosis and treatment initia-
Early diagnosis and treatment initiation could lead to improved survival in nonHIV PCP. tion could lead to improved survival in non-HIV PCP $(15,16,38)$. In other studies, it was reported that low serum albumin levels and mechanical ventilation were independent predictors of mortality by multivariate analyses $(40,41)$. In our study, performance status of $2-4$, treatment delay and high (severe) disease activity were unfavorable prognostic factors by an univariate analysis (42). More studies and analyses are needed.

\section{Conclusion}

While an emergence of non-HIV PCP has been increased with development of new immunosuppressive agents and biologics (4-8), there are many clinical issues to discuss regarding non-HIV PCP. Especially, approach to definite diagnosis and the quest for accurate treatment and chemoprophylaxis for non-HIV PCP should be discussed. Further studies are warranted and are believed to improve outcome of nonHIV PCP.

\section{Acknowledgement}

We are grateful for the diligent and thorough critical reading of our manuscript by Mr. John Wocher, Executive Vice President and Director, International Affairs/International Patient Services, Kameda Medical Center (Japan).

\section{References}

1. Thomas CF Jr, Limper AH. Pneumocystis pneumonia. N Engl J Med. 2004;350:2487-8.

2. Kovacs JA, Masur H. Evolving health effects of Pneumocystis: one hundred years of progress in diagnosis and treatment. JAMA. 2009;301:2578-85.

3. Limper AH, Offord KP, Smith TF, Martin WJ 2nd. Pneumocystis pneumonia. Differences in lung parasite number and inflammation in patients with and 
without AIDS. Am Rev Respir Dis. 1989;140:1204-9.

4. Koike $\mathrm{T}$, Harigai $\mathrm{M}$, Inokuma $\mathrm{S}$, Ishiguro $\mathrm{N}$, Ryu J, Takeuchi T, et al. Postmarketing surveillance of safety and effectiveness of etanercept in Japanese patients with rheumatoid arthritis. Mod Rheumatol. 2011; 21:343-51

5. Ward MM, Donald F. Pneumocystis carinii pneumonia in patients with connective tissue disease: the role of hospital experience in diagnosis and mortality. Arthritis Rheum. 1999;42:780-9.

6. Velayos FS, Sandborn WJ. Pneumocystis carinii pneumonia during maintenance anti-tumor necrosis factor-a therapy with infliximab for Crohn's disease. Inflamm Bowel Dis. 2004;10:657-60.

7. Seddik M, Meliez H, Seguy D, Viget N, Cortot A, Colombel JF. Pneumocystis jirovecii (carinii) pneumonia following initiation of infliximab and azathioprine therapy in a patient with Crohn's disease. Inflamm Bowel Dis. 2004;10:436-7.

8. Takeuchi T, Tatsuki Y, Nogami Y, Ishiguro N, Tanaka $\mathrm{Y}$, Yamanaka $\mathrm{H}$, et al. Postmarketing surveillance of the safety profile of infliximab in 5000 Japanese patients with rheumatoid arthritis. Ann Rheum Dis. 2008;67:189-4.

9. de Boer MG, Bruijnesteijn van Coppenraet LE, Gaasbeek A, Berger SP, Gelinck LB, van Houwelingen $\mathrm{HC}$, et al. An outbreak of Pneumocystis jiroveci pneumonia with 1 predominant genotype among renal transplant recipients: interhuman transmission or a common environmental source? Clin Infect Dis. 2007;44:1143-9.

10. Schmoldt S, Schuhegger R, Wendler T, Huber I, So"llner H, Hogardt M, et al. Molecular evidence of nosocomial Pneumocystis jirovecii transmission among 16 patients after kidney transplantation. J Clin Microbiol. 2008;46:966-71.

11. Radisic M, Lattes R, Chapman JF, del Carmen Rial M, Guardia O, Seu F, et al. Risk factors for Pneumocystis carinii pneumonia in kidney transplant recipients: a case-control study. Transpl Infect Dis. 2003; 5:84-93.

12. Sepkowitz KA, Brown AE, Telzak EE, Gottlieb S, Armstrong D. Pneumocystis carinii pneumonia among patients without AIDS at a cancer hospital. JAMA. 1992;267:832-7.

13. Barbounis V, Aperis G, Gambletsas E, Koumakis G, Demiris M, Vassilomanolakis M, et al. Pneumocystis carinii pneumonia in patients with solid tumors and lymphomas: predisposing factors and outcome. Anticancer Res. 2005;25:651-5.

14. Roux A, Canet E, Valade S, et al. Pneumocystis jirovecii pneumonia in patients with or without AIDS, France. Emerg Infect Dis. 2014;20:1490-7.

15. Asai N, Motojima S, Ohkuni Y, et al. Non-HIV Pneumocystis pneumonia: do conventional community-acquired pneumonia guidelines under estimate its severity? Multidiscip Respir Med. 2012;7:2.

16. Asai N, Motojima S, Ohkuni Y, et al. Early diagnosis and treatment are crucial for the survival of Pneumocystis pneumonia patients without human immunodeficiency virus infection. J Infect Chemother. 2012; 18:898-905.
17. Procop GW, et al. Detection of Pneumocystis jirovecii in respiratory specimens by four staining methods. $J$ Clin Microbiol. 2004;42:3333.

18. Mori S, Cho I, Sugimoto M. A follow up study of asymptomatic carriers of Pneumocystis jiroveci during immunosuppressive therapy for rheumatoid arthritis. J Rheumatol. 2009;36:1600-5.

19. Desmet S, Van Wijngaerden E, Maertens J, et al. Serum (1-3)-beta-D-glucan as a tool for diagnosis of Pneumocystis jirovecii pneumonia in patients with human immunodeficiency virus infection or hematological malignancy. J Clin Microbiol. 2009;47:3871-4.

20. Held J, Koch MS, Reischl U, et al. Serum $(1 \rightarrow 3)-\beta$ D-glucan measurement as an early indicator of Pneumocystis jirovecii pneumonia and evaluation of its prognostic value. Clin Microbiol Infect. 2011;17:595602.

21. Nakamura H, Tateyama M, Tasato D, et al. Clinical utility of serum beta-D-glucan and KL-6 levels in Pneumocystis jirovecii pneumonia. Intern Med. 2009;48:195-202

22. Hamada H, Kohno N, Yokoyama A, et al. KL-6 as a serologic indicator of Pneumocystis carinii pneumonia in immunocompromised hosts. Intern Med. 1998; 37:307-10.

23. Tasaka S, Hasegawa N, Kobayashi S, et al. Serum indicators for the diagnosis of pneumocystis pneumonia. Chest. 2007;131:1173-80.

24. Watanabe T, Yasuoka A, Tanuma J, et al. Serum (1$>3$ ) beta-D-glucan as a noninvasive adjunct marker for the diagnosis of Pneumocystis pneumonia in patients with AIDS. Clin Infect Dis. 2009;49:1128-31.

25. Esteves F, Calé SS, Badura R, et al. Diagnosis of Pneumocystis pneumonia: evaluation of four serologic biomarkers. Clin Microbiol Infect. 2015;21:379.

26. Asai N, Aoshima M, Ohkuni Y, Kobayashi H, Matsunuma R, Nakashima K, et al. A successful diagnostic case of Pneumocystis pneumonia by the loop-mediated isothermal amplification method in a patient with dermatomyositis. J Infect Chemother. 2012;18: 965-9.

27. Nakashima K, Aoshima M, Ohkuni Y, et al. Loop-mediated isothermal amplification method for diagnosing Pneumocystis pneumonia in HIV-uninfected immunocompromised patients with pulmonary infiltrates. J Infect Chemother. 2014;20:757-61.

28. Kawano S, Maeda T, Suzuki T, et al. Loop-mediated isothermal amplification with the Procedure for Ultra Rapid Extraction kit for the diagnosis of pneumocystis pneumonia. J Infect Chemother. 2015;21:224-6.

29. Walzer PD, Smulian AG. Pneumocystis species. In: Mandell GL, Bennett JE, et al. Mandell, Douglas, and Bennett's principles and practice of infectious diseases. 7th ed. Philadelphia: Churchill Livingstone/Elsevier; 2010:3377-3390.

30. National Comprehensive Cancer Network (NCCN) Clinical Practice Guidelines in Oncology. Prevention and treatment of cancer-related infections. Version 2.2014. http://www.nccn.org (Accessed on 20 May 2015).

31. Tomblyn M, Chiller T, Einsele H, et al. Guidelines for preventing infectious complications among hemato- 
poietic cell transplantation recipients: a global perspective. Biol Blood Marrow Transplant. 2009;15: 1143.

32. Martin SI, Fishman JA, AST Infectious Diseases Community of Practice. Pneumocystis pneumonia in solid organ transplantation. Am J Transplant. 2013;13 Suppl 4:272.

33. Vananuvat $P$, Suwannalai $P$, Sungkanuparph $S$, et al. Primary prophylaxis for Pneumocystis jirovecii pneumonia in patients with connective tissue diseases. Semin Arthritis Rheum. 2011;41:497-502.

34. Moosig F, Holle JU, Gross WL. Value of anti-infective chemoprophylaxis in primary systemic vasculitis: what is the evidence? Arthritis Res Ther. 2009;11: 253.

35. Lapraik C, Watts R, Bacon P, et al. BSR and BHPR guidelines for the management of adults with ANCA associated vasculitis. Rheumatology (Oxford). 2007;46:1615-6.

36. Hughes WT, Kuhn S, Chaudhary S, et al. Successful chemoprophylaxis for Pneumocystis carinii pneumonitis. N Engl J Med. 1977;297:1419-26.

37. Green H, Paul M, Vidal L, et al. Prophylaxis of Pneu- mocystis pneumonia in immunocompromised nonHIV-infected patients: systematic review and metaanalysis of randomized controlled trials. Mayo Clin Proc. 2007;82:1052-9.

38. Vanhems P, Toma E. Evaluation of a prognostic score. Pneumocystis carinii pneumonia in HIV-infected patients. Chest. 1995;107:107-12.

39. Kim SJ, Lee J, Cho YJ, et al. Prognostic factors of Pneumocystis jirovecii pneumonia in patients without HIV infection. J Infect. 2014;69:88-95.

40. Koga M, Koibuchi T, Kikuchi T, et al. Kinetics of serum $\beta$-D-glucan after Pneumocystis pneumonia treatment in patients with AIDS. Intern Med. 2011;50: 1397-401.

41. Matsumura $Y$, Shindo $Y$, linuma $Y$, et al. Clinical characteristics of Pneumocystis pneumonia in non-HIV patients and prognostic factors including microbiological genotypes. BMC Infect Dis. 2011;11:76.

42. Asai N, Ohkuni Y, Matsunuma R, et al. Clinical manifestations and prognostic factors of pneumocystis pneumonia without HIV infection. 2011 ERS Annual meeting abstracts, P2531. 